\title{
Guia prático de referências e recomendações nutricionais para indivíduos acometidos pelo coronavírus e para a população em isolamento social durante a pandemia: COVID-19
}

\author{
Practical guide for references and nutritional recommendations for individuals affected by the \\ coronavirus and for the population in social isolation during the pandemic: COVID-19
}

DOI: $10.37111 /$ braspenj.2020352012

Hadassa Hillary Novaes Pereira Rodrigues' Wanderson Hugo Drescher ${ }^{2}$

Ana Carolina Pinheiro Volp ${ }^{3}$

\section{Unitermos:}

Estado nutricional. Recomendações Nutricionais. Imunidade. Inflamação. Infecções por Coronavirus.

\section{Keywords:}

Nutritional status. Recommended Dietary Allowances. Immunity. Inflammation. Coronavirus Infections.

\section{Endereço para correspondência:}

Ana Carolina Pinheiro Volp

Av. Fernando Corrêa da Costa, 2367 - Boa Esperança

- Cuiabá, MT, Brasil - CEP 78060-900

E-mail: anavolp@gmail.com

\section{Submissão}

19 de abril de 2020

Aceito para publicação

24 de junho de 2020

\begin{abstract}
RESUMO
Introdução: Após um surto de pneumonia de causa desconhecida registrado na China, em dezembro de 2019, descobriu-se uma doença de origem zoonótica causada pelo vírus da síndrome respiratória aguda grave (SARS-CoV), que ficou amplamente conhecida como doença coronavirus disease 2019 (COVID-19). Dentre os acometidos, uma parcela necessita de hospitalização e o suporte nutricional se faz necessário. Entretanto, os que estão em isolamento social devem amplificar o seu estado nutricional, bem como a sua imunidade, buscando criar mais resistência, caso sejam acometidos. $O$ objetivo do cuidado é não sobrecarregar o sistema de saúde, para que, pouco a pouco, os necessitados tenham atendimento digno. Considerando a necessidade de uma maior munição aos profissionais da saúde e população em geral, e de forma simplificada frente à "chuva" de informações que todos estão recebendo, o objetivo do presente trabalho foi sumarizar e apresentar um guia prático de referências para a população em isolamento social durante a pandemia. Método: Foi realizada uma busca literária em referências já publicadas sobre $o$ assunto em websites científicos, incluindo os principais temas: estado nutricional, recomendações nutricionais, imunidade e coronavírus. Resultados: Incluiu-se, neste guia, os seguintes itens, apresentados de forma bem clara: orientações para a população que está em isolamento social; lactantes com COVID-19; imunomodulação; compras e no retorno para casa. Conclusão: O presente artigo trouxe uma sumarização do que se tem disponível sobre a temática na literatura até hoje. Acreditamos que este material possa auxiliar aos profissionais e à população em geral, em tempos de pandemia.
\end{abstract}

\section{ABSTRACT}

Introduction: After an outbreak of pneumonia caused by an unknown cause registered in China in December 2019, a disease of zoonotic origin was detected, caused by severe acute respiratory syndrome virus (SARS-CoV), which became known as coronavirus disease due to days sickness 2019 (COVID-19). Of those affected, a portion needs hospitalization and nutritional support is necessary. However, those who are in social isolation must amplify their nutritional status, as well as their immunity, in the process of creating more resistance if they are affected. The goal of care is not to overload the health system, so that little by little the needy have dignified care. Considering the need for greater ammunition for health professionals and the population in general, and in a simplified way in the face of the "rain" of information that everyone is receiving, the objective of the present work was to summarize and present a practical reference guide for the population in social isolation during the pandemic. Methods: A literary search was carried out on references already published on the subject on scientific websites, including the main themes: nutritional status, nutritional recommendations, immunity and coronavirus. Results: The following were included in this guide, presented very clearly: guidelines for the population that is in social isolation; lactating with COVID-19; immunomodulation; shopping and returning home. Conclusion: This article has summarized what is available on the subject in the literature until today. We believe that this material can help professionals and the general population in times of pandemic.

1. Mestranda pelo Programa de Pós-Graduação em Ciências da Saúde, Universidade Federal de Mato Grosso, Faculdade de Medicina, Cuiabá, MT, Brasil.

2. Mestrando pelo Programa de Pós Graduação em Nutrição, Alimentos e Metabolismo, Universidade Federal de Mato Grosso, Faculdade de Nutrição, Cuiabá, MT, Brasil.

3. Doutora em Ciência e Tecnologia de Alimentos, Professora Associada da Universidade Federal de Mato Grosso, Faculdade de Nutrição, Departamento de Nutrição e Alimentos, Cuiabá, MT, Brasil. 


\section{INTRODUÇÃO}

Em dezembro de 2019, várias unidades de saúde em Wuhan, na China, registraram um surto de pneumonia de causa desconhecida. Posteriormente, descobriu-se que a então doença é causada pelo vírus da síndrome respiratória aguda grave (SARS-CoV-2), de origem zoonótica, - qual se tornou amplamente conhecido como novo coronavírus e coronavirus disease 2019 (COVID-19). Apresenta disseminação rápida de maneira exponencial e, atualmente, cursa como pandemia no mundo inteiro e vem causando colapso nos sistemas de saúde devido ao curto tempo de contágio e à necessidade de hospitalização e cuidados intensivos'.

Atualmente, são 9.015.582 milhões de casos confirmados em todo o mundo, sendo os maiores epicentros da doença os Estados Unidos, com 2.298.341 milhões de casos, o Brasil, com 1.083.341 milhões de casos, e a Rússia, com 591.465 mil de casos². O Brasil cursa com 50.591 mil óbitos, sendo as grandes metrópoles São Paulo e Rio de Janeiro as mais afetadas ${ }^{3}$. Ressalta-se que estes dados são dinâmicos e se alteram todos os dias. O Ministério da Saúde segue preconizando medidas de isolamento e distanciamento social e é apoiado por decretos estaduais e municipais brasileiros ${ }^{4}$. Ainda não há evidências científicas robustas quanto a medicações e/ou cura eficazes para a doença ${ }^{4}$.

Dentre os principais sintomas da COVID-19 estão a febre, a falta de ar e a sensação de cansaço. $O$ período de incubação é de 4 dias, variando de 2 a 7 dias. Cerca de $80 \%$ a $85 \%$ dos contaminados apresentam a forma leve da doença, entretanto, o restante necessita de hospitalização, sendo a maioria idosos e/ou portadores de comorbidades (cardiopatias, doença renal crônica, doenças respiratórias e diabetes) ${ }^{4}$.

O enfrentamento da pandemia do coronavírus está exigindo adaptações da população em geral, como o isolamento social e as medidas de proteção durante as compras nos supermercados. Tudo isto impacta diretamente nos níveis de estresse, ansiedade, alterações no ritmo do sono, qualidade e quantidade das refeições, bem como no padrão alimentar ${ }^{5}$. Além disso, é importante reiterar tudo que se conhece na literatura a respeito de uma alimentação saudável e adequada para todos, com referências atualizadas e compiladas de fácil acesso para os profissionais de linha de frente e também para a população. No presente trabalho, centra-se a atenção no cuidado e manutenção do estado nutricional, com recomendações nutricionais e orientações alimentares para diferentes grupos de populações, mas também sem esquecer daqueles que tiveram a doença, e agora curados, precisam de reabilitação funcional e manutenção do estado nutricional ${ }^{5}$.
A perda progressiva e/ou generalizada de massa muscular ou sarcopenia primária, muito presente nos idosos, é outro aspecto relevante do estado nutricional, e que ainda pode contribuir negativamente para o prognóstico daqueles infectados pelo coronavirus que precisarão de hospitalização $0^{6,7}$. Estes, na maioria das vezes, são portadores de comorbidades ou idosos que, posteriormente, passarão pela síndrome "post intensive care", caracterizada por alterações físicas, cognitivas e, principalmente, de perda da musculatura esquelética, estando associada à fragilidade e à redução da qualidade de vida .

Diante do exposto, é essencial que ações de caráter assistenciais e emergenciais por parte da nutrição sejam substanciadas e referenciadas para a facilidade de acesso à população em geral e também aos profissionais que estão na linha de frente no combate ao coronavirus.

Desta forma, dada a relevância clínica e de saúde pública da temática em questão, o objetivo do presente artigo é sumarizar e compilar as principais referências em recomendações nutricionais durante o enfrentamento da COVID-19 na forma de orientações nutricionais a toda população, bem como medidas de proteção para sair de casa.

\section{MÉTODO}

Este estudo consiste em revisão bibliográfica de artigos contendo estudos nacionais e internacionais que relacionam a temática principal. A presente revisão bibliográfica da literatura foi realizada a partir dos periódicos disponíveis no portal da Coordenação de Aperfeiçoamento de Pessoal de Nível Superior (CAPES) e nas principais bases de dados em saúde: MEDLINE, LILACS, PubMed e SciELO. A sistematização utilizada incluiu a análise e interpretação de material que contivesse "coronavirus" mais qualquer uma das palavras chaves citadas (estado nutricional, recomendações nutricionais, imunidade e inflamação).

\section{RESULTADOS}

Os mesmos serão apresentados de forma sucinta e clara, na seguinte sequência: orientações para a população que está em isolamento social, lactantes com COVID-19, alimentação imunomoduladora, medidas de proteção para durante as compras e no retorno para casa.

\section{Recomendações Dietéticas e Nutricionais}

\section{Para a População em Geral}

Conforme as recomendações baseadas no Guia alimentar para população brasileira de 20148 , sugere-se 
que a população em geral continue realizando as seguintes ações, porém tomando como cuidado as seguintes premissas:

- Compre de pequenos produtores/negócios, a alimentação saudável deriva de um sistema alimentar socialmente e ambientalmente sustentável, levando em conta o impacto das formas de produção e distribuição dos alimentos, sobre a justiça social e ambiental ${ }^{8}$;

- Faça dos alimentos in natura ou minimamente processados a base da sua alimentação: legumes, verduras, frutas, frutas secas, sucos de frutas, cerais, raízes e tubérculos, oleaginosas, farinhas, carnes, laticínios e ovos ${ }^{8}$;

- Utilize sal, açúcar, óleos e gorduras em pequenas quantidades no preparo das refeições ${ }^{8}$;

- Limite o consumo de alimentos processados (alimentos conservados em salmoura, concentrados com sal e/ ou açúcar). No processamento em geral, são utilizadas quantidades muito superiores às usadas em preparações culinárias, alteram de modo desfavorável a composição nutricional dos alimentos ${ }^{8}$;

- Evite o consumo de alimentos ultraprocessados, pois são nutricionalmente desbalanceados, ricos em calorias vazias e aumentam suas despesas. Envolvem diversas etapas de processamento e muitos ingredientes, incluindo sal, açúcar, óleos e gorduras e substância exclusivas de uso industrial. São exemplos: guloseimas e bebidas adoçadas com açúcar ou adoçantes artificiais, refrescos em pó, embutidos derivados de carne e gordura animal, produtos congelados prontos para aquecer, misturas para bolos, sopas em pó, macarrão instantâneo, temperos prontos, salgadinhos de pacotes, biscoitos recheados, entre outros ${ }^{8,9}$;

- Minimize a ida aos centros de abastecimento, como mercados e supermercados, postos de combustíveis, farmácias, entre outros, durante a pandemia, mas, mesmo assim, procure seguir se alimentando de forma saudávelp;

- Somente saia de casa em extrema necessidade, faça uma lista de compras e use-a. Você pode esquecer alguns itens ou comprar outros por impulso";

- Faça compras em horários alternativos ou no sistema online, no qual são adotadas medidas de controle e proteção. Você economizará tempo e manterá a distância social. Não deixe de comprar com antecedência, pois muitas lojas precisam de um ou dois dias desde o pedido até a entrega";

- Considere alternativas de baixo custo e faça o consumo de alimentos de forma integral, utilizando talos, cascas e sementes ${ }^{9}$;

- Planeje o uso do seu tempo para dar à alimentação o espaço que ela merece: organize uma lista de compras, organize a despensa doméstica e/ou a cozinha, defina o cardápio da semana, reavalie como você tem usado seu tempo e identifique quais atividades poderiam ceder espaço para a alimentação ${ }^{8}$;

- Hidrate-se: a água é essencial para a manutenção de todos os processos do corpo. O total de água no corpo humano corresponde a mais da metade do peso na idade adulta, e a água pura, potável, é a melhor opção para ingestão de líquidos ${ }^{8}$;

- Mantenha-se fisicamente ativo. A atividade física regular é importante para evitar condições crônicas de saúde, como ganho de peso, perda de massa e força muscular e perda da competência imune do organismo ${ }^{7}$.

\section{Lactantes com COVID-19}

Sabidamente, o aleitamento materno confere proteção contra muitas doenças e são raras as exceções em que não é recomendado. Uma vez que a mãe seja esclarecida e esteja de acordo, é possível manter o aleitamento materno mesmo em caso de contaminação da mãe, conforme precauções necessárias: uso de máscara pela lactante e lavagem de mãos antes das mamadas. Portanto, salvo alguma intercorrência que exija a separação lactante e lactente, como, por exemplo, o agravamento das condições de saúde maternas, é perfeitamente possível que mãe e filho permaneçam juntos em domićlio ou no ambiente hospitalar, caso seja necessário ${ }^{10,11}$.

Caso a mulher não se sinta segura em amamentar enquanto estiver com coronavírus, recomenda-se que seu leite seja retirado e ofertado à criança. Tendo em vista que a mãe infectada pode transmitir o vírus por meio de gotículas respiratórias, são recomendadas as seguintes orientações para as lactantes infectadas pelo vírus ${ }^{10,11}$ :

- Lavar as mãos por, pelo menos, 20 segundos antes de tocar o bebê ou antes de retirar o leite materno (extração manual ou na bomba extratora);

- Usar máscara facial (cobrindo completamente nariz e boca), durante as mamadas, e evitar falar ou tossir durante a amamentação;

- A máscara deve ser imediatamente trocada em caso de tosse, espirro ou a cada nova mamada, com nova higienização das mãos após a troca;

- Seguir rigorosamente as recomendações para limpeza das bombas de extração de leite após cada uso;

- Deve-se considerar a possibilidade de solicitar a ajuda de alguém que esteja saudável para oferecer o leite materno em copinho, xícara ou colher ao bebê. É necessário que a pessoa que vai oferecer ao bebê aprenda a fazer isso com a ajuda de um profissional de saúde. 


\section{Fortalecimento do Sistema Imunológico}

- A alimentação ideal e equilibrada deve fornecer em quantidade e qualidade todos os nutrientes necessários para a manutenção do estado nutricional adequado, nos diversos ciclos da vida e no bom funcionamento do organismo, em especial, o sistema imunológico ${ }^{12-14}$;

- A necessidade de suplementação de qualquer nutriente deve ser avaliada pelo profissional adequado, de acordo com o resultado de exames laboratoriais e recomendações de diretrizes de tratamento, a fim de evitar super dosagens e interações com outros nutrientes que prejudiquem sua absorção ${ }^{13,14}$;

Seguem alguns nutrientes e suas fontes alimentares que podem dar suporte a nossa imunidade:

- Ferro: este nutriente compõe a estrutura de várias proteínas, entre elas enzimas, mioglobina e hemoglobina, entre outras. Atualmente, alguns estudos têm demonstrado a associação da deficiência de ferro a defeitos na resposta imunitária adaptativa e também na resposta inata do indivíduo, sendo esta a primeira linha de defesa. A forma orgânica conhecida como ferro heme, que é mais absorvível pelo organismo, está presente em alimentos como carnes vermelhas, peixes e aves. Em ovos, frutas secas, feijões, castanhas, vegetais de folhas verdes escuras, principalmente, quinoa, grão de bico, lentilha, entre outros cereais, está presente o ferro não heme, que, por sua vez, sofre influência de inibidores ou amplificadores da absorção pelo organismo. A combinação do consumo de alimentos ricos em vitamina $C$ com fontes alimentares de ferro, melhoram a absorção destes nutrientes ${ }^{13,14}$;

- Zinco: este mineral é encontrado em sementes, castanhas, amêndoas, cereais integrais, casca de tangerina, carnes e laticínios. Possui diversas atividades biológicas, como, por exemplo, parte integrante de enzimas antioxidantes e tem papel importante na relação com células do sistema imunitário, como atividade de células T auxiliadoras, retardamento da hipersensibilidade, proliferação de linfócitos T, produção de interleucina-2 entre outros ${ }^{13,14}$;

- Vitamina A: sua participação no sistema imunológico, assim como de seus metabólitos, como o ácido retinóico, e carotenoides, como o ß-caroteno, é descrita na literatura por desempenhar papel estimulando a fagocitose, aumento na resposta de timócitos a mitógenos específicos, proporciona a liberação seletiva de Interleucina- 1 por monócitos, entre outros. Suas fontes alimentares são cenoura, batata doce, folhas de brócolis, manga, couve, fígado, peixe, leite e derivados ${ }^{13,14}$;

- Vitamina E: Sua deficiência está relacionada com o comprometimento de vários aspectos da resposta imune, entre elas, mediadas por células B e T. Apresenta ação antioxidante. Suas fontes alimentares são óleos vegetais, como azeite de oliva extra virgem, germe de trigo, milho, soja, castanhas, avelã, semente de girassol e abacate ${ }^{13,14}$;

- Vitamina C: Está presente em frutas e legumes, como kiwi, goiaba, brócolis, frutas cítricas, frutas vermelhas, talos de couve e salsa. É encontrada em grande concentração em células do sistema imune. Apresenta atividade antioxidante, protegendo as células de danos oxidativos. Auxilia nas funções dos fagócitos, proliferação de linfócitos T e expressão gênica das moléculas de adesão dos monócitos e na produção de citocinas ${ }^{13,14}$;

- Vitamina D: O papel da Vitamina D na regulação do cálcio sérico e saúde óssea já é bem conhecido, atualmente vem se destacando também pelo seu papel na manutenção do sistema imunológico. A deficiência dessa vitamina tem sido relatada em várias condições, como no aumento da inflamação do organismo e na desregulação do sistema imunológico por meio de dois pontos importantes: o receptor de vitamina $D(V D R)$ é expresso pelas maiorias das células imunes e a sinalização dessa vitamina e VDR, em conjunto, possuem efeito anti-inflamatório e supressor na autoimunidade. Estudos recentes apontam que a suplementação precoce de vitamina $D$ apresenta efeito protetor em casos não críticos de COVID-19, e que este efeito foi maior em indivíduos com a deficiência dessa vitamina que receberam a suplementação. Há um número crescente de evidências que indicam a melhora da recuperação imunológica durante a terapia retroviral e aumento da imunidade contra patógenos com a suplementação desta vitamina. Alguns mecanismos fisiológicos explicam este efeito, entre eles a indução de catelicidinas e defensinas, peptídeos que podem reduzir as taxas de replicação viral e diminuição da inflamação. A exposição solar é forma mais eficiente para produção de vitamina $D$, o consumo de alguns peixes e ovos podem ser aliados ${ }^{13-17}$;

- Ômega-6 e ômega-3: Genericamente, denominados de ácidos graxos poli-insaturados, ambos são considerados essenciais ao organismo e participam de diversas funções orgânicas, como manter membranas celulares, funções cerebrais e transmissão de impulsos nervosos. Ainda, estão diretamente ligados ao sistema imune e à resposta inflamatória, uma vez que são precursores na síntese de eicosanoides (prostaglandinas e leucotrienos). Os eicosanoides derivados do ômega-3 possuem características anti-inflamatórias e os derivados do ômega-6 características inflamatórias, quando em excesso no organismo. Por isso, são propostas razões para o consumo equilibrado entre ômega-6:ômega-3, a Organização Mundial de Saúde/ The Food and Agriculture Organization determina a razão de 5-10:1. Principais fontes alimentares de ômega-3 são peixes marinhos (salmão, sardinha, atum entre outros), linhaça, castanhas, nozes, sementes 
entre outros, e fontes alimentares de ômega-6 são óleos vegetais (milho, soja, girassol, entre outros) ${ }^{13,18-20}$;

- Prebióticos: são partes não-digeríveis de carboidratos, as quais estimulam o crescimento e/ou atividade de bactérias no colo, tendo resultados benéficos à saúde, na prevenção das doenças alérgicas e modulando a resposta imunológica, auxiliando na diferenciação de células de defesa. Alimentos ricos em prebióticos são cebola, alho, farinha de banana verde, batata doce, batata yacon, além de fibras encontradas em cereais integrais, frutas, verduras, legumes e sementes ${ }^{21}$;

- Probióticos: também são descritos efeitos benéficos no sistema imunológico para este grupo, uma vez que são organismos vivos que compõem o microbioma do trato gastrintestinal, produzindo efeitos benéficos quando em quantidades adequadas. Promovem a saúde intestinal juntamente com os prebióticos e, ainda, promovem a manutenção e reparo na barreira intestinal, efeitos antiinflamatórios, auxiliam na resposta imunológica inata e diferenciação de células de defesa, entre outros. Alguns alimentos são compostos por probióticos, como leites fermentados e iogurtes ${ }^{21}$;

- Pigmentos naturais: estão presentes não apenas para colorir os alimentos, mas também apresentam ações benéficas ao organismo, como antioxidantes e têm sido associados à promoção da saúde e à prevenção de doenças crônicas. De maneira geral, orienta-se que a alimentação seja a mais colorida possível, para fornecer uma gama de compostos bioativos para desempenharem suas funções orgânicas. Estão presentes em diversos alimentos, como frutas, verduras e legumes em geral ${ }^{22}$.

Cabe ressaltar que um único alimento/nutriente não apresenta este efeito, e sim, é preciso ter uma alimentação equilibrada, juntamente com o estilo de vida saudável.

\section{Na Hora das Compras e do Retorno para Casa}

- Caso você pertença ao grupo de risco ou apresente quaisquer sintomas de doenças respiratórias, não vá às compras ${ }^{9,23}$;

- Planejar o que irá consumir para 2 semanas para minimizar as idas ao supermercado 9,23 ;

- Limpar o carrinho do supermercado com álcool 70\%, antes de colocar as mãos ou as compras ${ }^{9,23}$;

- Guarde álcool em gel ou líquido 70\% com um rolo de papel higiênico ou papel toalha no carro e um saquinho para descartes ${ }^{9,23}$;

- Molhe o papel com álcool e passe no volante, freio de mão, alavanca de marchas, maçaneta interna das portas, controle de vidros e controles de rádio ${ }^{9,23}$;

- Considere sua mão sempre suja, nunca a leve até a boca, nariz ou olhos ${ }^{9,23}$;
- Evite ficar a menos de 1 metro de outra pessoa ${ }^{9,23}$;

- Sempre que chegar em casa tire os sapatos, troque de roupa, lave as mãos e os braços até os cotovelos ${ }^{9,23}$;

- Passe o álcool 70\% ou lave com água e sabão se possível, 1 vez por dia em torneiras, maçanetas das portas, chaves de casa e do carro, celular e tablete, notebook, computadores, teclado, mouse e controles remotos ${ }^{9,23}$;

- Lavar as mãos antes de manipular os alimentos, evitar tossir ou espirrar sobre eles ${ }^{9,23}$;

- Em casa, é importante ter um local específico para colocar todas as compras, por exemplo, uma mesa. Divida este local em duas áreas: área limpa e área suja. Higienize o local com qualquer produto desinfetante ou sanitizante que tenha em $\operatorname{casa}^{9,23}$;

- Na área suja, coloque todas as sacolas que acabaram de chegar do mercado 9,23;

- Retire os alimentos e produtos das sacolas e à medida que limpa suas embalagens, coloque-as na área limpa ${ }^{9,23}$;

- Vidros, latas e plásticos rígidos podem ser lavados com água e sabão ${ }^{23}$;

- Embalagens flexíveis podem ser limpas com papel toalha e álcool $70 \%{ }^{23}$;

- Frutas verduras e legumes, se forem armazenadas sob refrigeração, devem ser lavados e higienizados com solução de hipoclorito de sódio antes de serem armazenados. A proporção e tempo de uso vem indicado na embalagem de cada marca comercial. Caso for armazenar em fruteira, não lave antes de colocar na mesma, lavar somente antes do consumo 9,23 ;

- Alimentos com duas embalagens, descarte a embalagem externa antes de ser armazenado 9,23 ;

- Branqueamento é uma técnica de conservação para legumes e folhas, o qual reduz a carga microbiana, ação enzimática natural de amadurecimento (processamento térmico) e aumentando a durabilidade. Nada mais é que porcioná-los, colocá-los em água fervente de 1 a 3 minutos (quando mais consistente o alimento, maior o tempo) e depois mergulhá-los imediatamente na água bem fria pelo mesmo período, dando o choque térmico e preservando, assim, o valor nutricional, coloração e o sabor ${ }^{9,23}$.

\section{CONCLUSÃO}

Os resultados apresentados nesta revisão apresentam importância clínica relevante. O presente artigo trouxe uma sumarização do que se tem disponível sobre a nova temática existente na literatura até hoje. Acreditamos que este material possa auxiliar aos profissionais e à população em geral, em tempos de pandemia, e que possamos passar por ela da maneira mais segura possível. 


\section{REFERÊNCIAS}

1. Wu Z, McGoogan JM. Characteristics of and important lessons from the coronavirus disease 2019 (COVID-19) outbreak in China: summary of a report of 72,314 cases from the Chinese Center for Disease Control and Prevention. JAMA. 2020; 10.1001/jama.2020.2648.

2. Johns Hopkins University. COVID-19 dashboard by the Center for Systems Science and Engineering (CSSE) at Johns Hopkins University (JHU). [cited 2020 Apr 16]. Available from: https://www.arcgis.com/apps/opsdashboard/index.html\#/ bda7594740fd40299423467b48e9ecf6

3. Brasil. Ministério da Saúde. COVID-19 no Brasil. [cited 2020 Apr 16]. Available from: http://susanalitico.saude.gov.br/\#/ dashboard/

4. Parra BFCS, Matos LBN, Ferrer R, Toledo DO. SARCPRO: proposta de protocolo para sarcopenia em pacientes internados. BRASPEN J. 2019;34(1):58-63.

5. American Society of Nutrition. Making health and nutrition a priority during the coronavirus (COVID-19) pandemic. [cited 2020 Apr 16]. Available from: https://nutrition.org/makinghealth-and-nutrition-a-priority-during-the-coronavirus-covid19-pandemic/

6. Campos ACL, Matsuba CST, Aanholt DPJV, Nunes DSL, Toledo DO, Rocha EEM, et al. Diretriz brasileira de terapia nutricional no paciente grave. BRASPEN J. 2018;33(supl. 1):2-36.

7. Barazzoni R, Bischoff SC, Breda J, Wickramasinghe K, Krznaric Z, Nitzan D, et al. ESPEN expert statements and practical guidance for nutritional management of individuals with SARS-CoV-2 infection. Clin Nutr. 2020;39(6): 1631-8.

8. Brasil. Ministério da Saúde. Guia alimentar para população brasileira. 2a ed. Brasília: Ministério da Saúde; 2014.

9. Ros DC, Brasioli M, Guilherme RC. Guia para uma alimentação saudável em tempos de COVID-19. São Paulo: Associação Brasileira de Nutrição - ASBRAN; 2020.

10. Brasil. Ministério da Saúde, Secretaria de Atenção Primária à Saúde. Nota técnica No 7/2020-DAPES/SAPS/MS. Brasília: Ministério da Saúde; 2020.

11. Royal College of Obstetricians and Gynaecologists. Coronavirus (COVID-19) infection and pregnancy. Information for healthcare professionals: Version 8. London: Royal College of Obstetricians and Gynaecologists; 2020.

12. Cruz-Jentoft AJ, Bahat G, Bauer J, Boirie Y, Bruyère O, Cederholm T, et al. Sarcopenia: revised European consensus on definition and diagnosis. Age Ageing. 2019;48(1):16-31.

13. Biasebetti MBC, Rodrigues ID, Mazur CE. Relação do consumo de vitaminas e minerais com o sistema imunitário: uma breve revisão. Visão Acadêmica. 2018;19(1):130-6.

14. Sarni ROS, Souza FIS, Cocco RR, Mallozi MC, Solé D. Micronutrientes e sistema imunológico. Rev Bras Alerg Imunopatol. 2010;33(1):8-13.

15. Sassi F, Tamone C, D'Amelio P. Vitamin D: nutrient, hormone, and immunomodulator. Nutrients. 2018;10(11):1656.

16. Grant WB, Lahore H, McDonnell SL, Baggerly CA, French CB, Aliano JL, et al. Evidence that vitamin D supplementation could reduce risk of influenza and COVID-19 infections and deaths. Nutrients. 2020;12(4):988.

17. Caccialanza R, Laviano A, Lobascio F, Montagna E, Bruno R, Ludovisi S, et al. Early nutritional supplementation in noncritically ill patients hospitalized for the 2019 novel coronavirus disease (COVID-19): rationale and feasibility of a shared pragmatic protocol. Nutrition. 2020;74:1108353.

18. Martin CA, Almeida VV, Ruiz MR, Visentainer JEL, Matshushita M, Souza NE, et al. Ácidos graxos poliinsaturados ômega-3 e ômega-6: importância e ocorrência em alimentos. Rev Nutr. 2006;19(6):761-70.

19. Perini JAL, Stevanato FB, Sargi SC, Visentainer JEL, Dalalio MMO, Matshushita M, et al. Ácidos graxos poli-insaturados n-3 e n-6: metabolismo em mamíferos e resposta imune. Rev Nutr. 2010;23(6):1075-86.

20. World Health Organization and FAO Joint Consultation. Fats and oils in human nutrition. Nutr Rev. 1995;53(7):202-5.

21. Souza FS, Cocco RR, Sarni ROS, Mallozi MC, Solé D. Prebiotics, probiotics and symbiotics on prevention and treatment of allergic diseases. Rev Paul Pediatr. 2010;28(1):86-97.

22. Reis RC, Viana ES, Jesus JL, Lima LF, Neves TT, Conceição EA. Compostos bioativos e atividade antioxidante de variedades melhoradas de mamão. Ciênc Rural. 2015;45(11):2076-81.

23. Food Safety Brazil. Comprando alimentos durante a pandemia de COVID-19. [cited 2020 Apr 10]. Available from: https://foodsafetybrazil.org/ comprando-alimentos-durante-pandemia-de-covid-19/

Local de realização do estudo: Universidade Federal de Mato Grosso, Cuiabá, MT, Brasil.

Conflito de interesse: Os autores declaram não haver. 